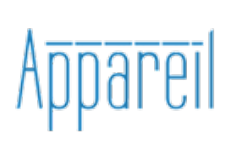

Appareil

14 | 2014

Esthétiques latino-américaines : penser à rebours

\title{
La question du son et l'Amérique latine : au-delà des cartographies
}

Gustavo Celedón Bórquez

\section{(2) OpenEdition}

1 Journals

Édition électronique

URL : http://journals.openedition.org/appareil/2102

DOI : 10.4000/appareil.2102

ISSN : 2101-0714

Éditeur

MSH Paris Nord

Référence électronique

Gustavo Celedón Bórquez, "La question du son et l'Amérique latine : au-delà des cartographies », Appareil [En ligne], 14 | 2014, mis en ligne le 10 décembre 2014, consulté le 10 décembre 2020. URL: http://journals.openedition.org/appareil/2102 ; DOI : https://doi.org/10.4000/appareil.2102

Ce document a été généré automatiquement le 10 décembre 2020.

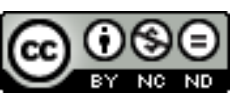

Appareil est mis à disposition selon les termes de la Licence Creative Commons Attribution - Pas d'Utilisation Commerciale - Pas de Modification 4.0 International. 


\section{La question du son et l'Amérique latine : au-delà des cartographies}

\section{Gustavo Celedón Bórquez}

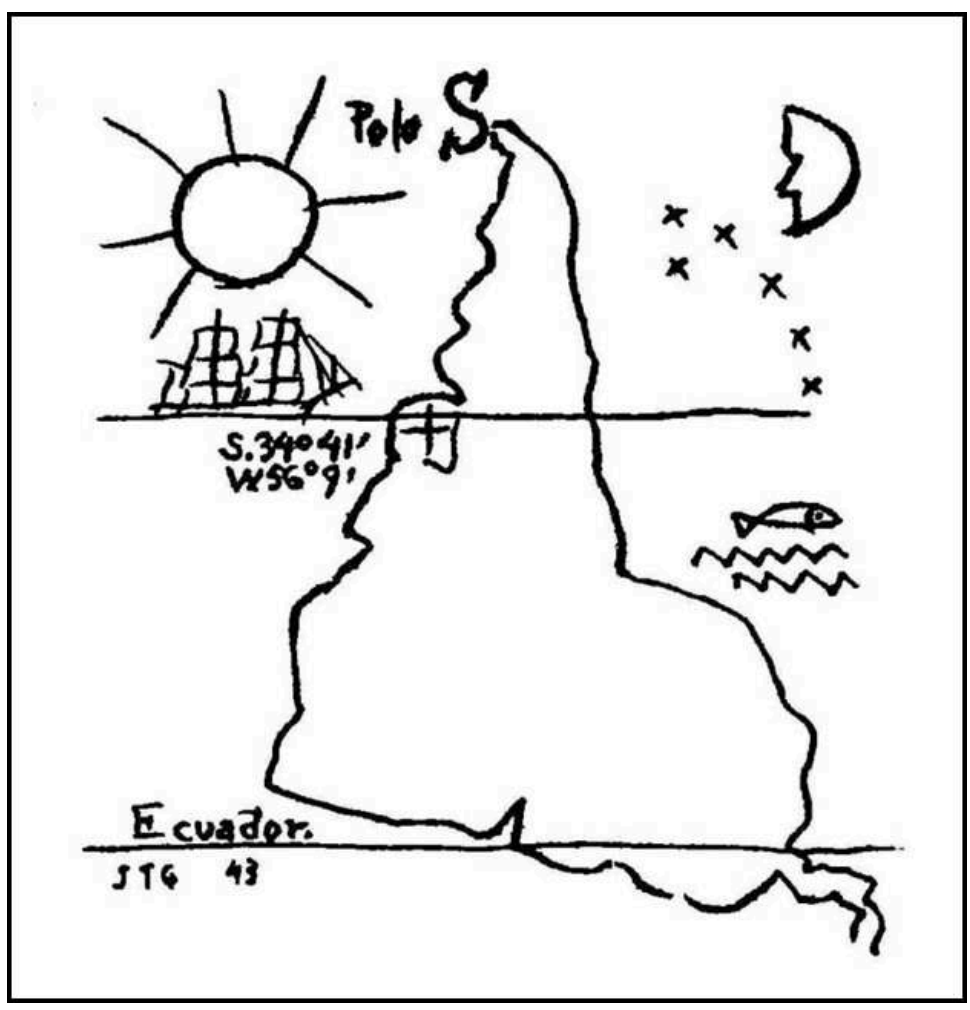

1 Cette image de l'Amérique latine mise à l'envers est très connue. Il s'agit d'un dessin de 1943 du peintre uruguayen Joaquín Torres García dont le titre est justement América Invertida (l'Amérique inversée). Aujourd'hui on peut la trouver à différents endroits, comme, par exemple, sur le logo du Centro de Estudios del Pensamiento Latinoamericano (CEPLA, Centre d'études de la pensée latino-américaine) de l'Université de Playa Ancha de Valparaíso, au Chili ${ }^{1}$. La consigne du peintre était « notre nord est le Sud $»^{2}$. 
J'ai dit École du Sud car en vérité notre nord est le Sud. Il ne doit pas y avoir le nord pour nous mais par opposition au Sud.

C'est pourquoi maintenant nous mettons la carte à l'envers, et donc nous avons déjà une juste idée de notre position et non comme ils veulent dans le reste du monde. La pointe de l'Amérique, dès lors, tout en se prolongeant, marque instamment le Sud, notre nord. De même, notre boussole : elle s'incline irrémissiblement toujours vers le Sud, jusqu'à notre pôle. Les bateaux, lorsqu'ils partent d'ici, descendent, ne montent pas, comme auparavant, pour s'en aller au nord. Parce que le nord est maintenant dessous. Et le Levant, en nous plaçant face à notre Sud, est à notre gauche.

Cette rectification était nécessaire ; grâce à elle, nous savons maintenant où nous sommes. ${ }^{3}$

2 Il faut poser la question suivante : s'agit-il d'une question de cartes ? Est-ce une affaire de cartographie?

3 Avant tout, il semble y avoir un repositionnement du sensible, la constatation d'un développement des sens qui ne s'orientent pas à la manière dont la cartographie mondiale a dessiné non seulement l'Orient et la position de l'Amérique du Sud, mais surtout la direction dont les sens d'une Amérique latine doivent être constamment guidés. La carte a toujours assuré un sens des sens, le sens du monde et le monde comme forme du - des - sens.

Mais l'inversion de Torres García n'est pas une affaire cartographique. Pas simplement. Il ne fait pas une carte. Il fait un dessin, une œuvre d'art si vous voulez. Cette différence est importante: le dessin agit comme une sorte d'articulation sensible dénonçant la postérité de toute carte, le contrat sensible que cette dernière a mis en marche. Le territoire est en quelque sorte fixé avant sa réalisation. Cette fixation est une fixation des sens : on a déjà un Orient, on sait déjà que le monde est rond, on sait où est le roi et on sait d'où Dieu nous regarde. On pourra dire ainsi : la carte est la confirmation d'une territorialisation, l'articulation d'une articulation, l'appareil d'un appareil, la coordination des coordonnés sensibles qui orientaient, avant la carte, le dessin des coordonnés de l'esprit, de l'idée qu'on a de l'espace.

Malgré les apparences, nous ne nous inscrivons pas dans l'idée d'une extériorisation technique de l'esprit humain qui, ensuite, se laisse former et déterminer par les spécificités propres à la technique à laquelle il s'est confié. Autrement dit : il n'y a pas une primauté de l'esprit à son extériorisation - il n'y a pas un désir d'extériorisation -, une âme qui, tout en s'extériorisant, matérialise sa mémoire et se donne forme ${ }^{4}$. Il n'y a pas non plus une extériorisation de l'esprit depuis les techniques, comme si l'esprit - ou l'idée, le sujet, la substance - était une sorte de fréquence ou émission des artefacts technologiques (comme si le territoire naissait parce qu'il y a des cartes). Certes, le topos uranos de Platon n'est pas le même lorsqu'il est envahi par des ondes de satellite, wifi, antennes, signaux de télécommunication : l'âme, l'Idée ou le noûs devront toujours leur forme à divers types de technification du ciel, de l'ambiance, de l'entourage. Mais on soutiendra la thèse suivante : il n'y a pas une antériorité ou une extériorité. Il y a plutôt une différance, au sens derridien, où l'esprit et la technique sont plongés dans un mélange d'événements sans porteur, sans pouvoir tracer la ligne exacte d'une séparation ou le temps précise d'une antériorité.

La séparation qui fait Joaquín Torres García entre le dessin d'un côté et la carte de l'autre, n'est pas absolue. On pourra dire, en fait, qu'il y a une séparation entre l'artefact dessinateur et l'artefact cartographique, séparation qu'ont remarquée 
plusieurs artistes et théoriciens depuis quelques décennies, comme Jaspers Johns ${ }^{5}$ ou, récemment, Dennis Wood ${ }^{6}$. Mais une telle séparation pose la question de l'artefactualité qui les aurait maintenues ensemble et dont la séparation agirait comme une sorte de vide artefactuel, comme une sorte d'abîme qui entoure et pénètre l'artefactualité même. Quel est le dernier artefact? Quel est le secret d'un artefact? Ici s'ouvre en effet la question de l'espace où se place un artefact, de la ligne jamais assurée entre l'espace qu'il crée et la place qu'il y prend. Car un artefact ne peut se détacher de sa matérialité : il sera toujours par rapport à une matière qui l'excède ou qui ne le constitue pas. Ainsi, la construction de l'espace et le monde qu'il ouvre en tant qu'artefact, se placent sur une matérialité étrangère à cet espace même, sur une dimension de la matière qui peut même devenir tout à fait étrangère à la notion de monde.

7 Il y a un espace ou une dimension qui excèdent la cartographie, quelque chose qui n'est pas dans le monde ou par le monde. C'est cela l'América Invertida, c'est-à-dire le mouvement par lequel la carte est déplacée au-delà de l'espace et l'esprit que cette dernière contient, a construit, soutient, supporte et guide, conserve et établit.

C'est ainsi que le dessin de Joaquín Torres García fait d'abord la critique de l'espace comme monde, comme ce monde coordonné conforme au sens européen du monde et de l'espace - la rotondité du monde et l'axe Nord-Sud sur lequel la Terre roule - dont la carte est la réalité architecturale, l'orientation-même de toute expérience dans la Terre, la fixation de l'expérience comme expérience-du-monde et, ainsi, la propagation - pour reprendre la notion de Jacques Rancière - d'un partage déterminé du sensible ${ }^{7}$. Ainsi, il fait aussi une décoordination des sens, il les laisse sans Orient - car nous ne saurons pas encore ce qui est le Sud, ce qui est « notre position $»^{8}$.

Cela veut dire que le renversement de l'América Invertida verse les sens de l'autre côté, comme si d'emblée le regard, l'écoute et l'odorat tombaient vers une direction nouvelle, inconnue, contraire au devenir du monde et, pourquoi pas, contraire au devenir même. Il ne s'agit pas non plus de l'identité : dans le Sud on ne trouve pas la vraie identité de l'Amérique latine. Ce qu'il y a là, dans le Sud, vers le Sud, c'est un détachement des sens de la direction générale du monde et même de la direction comme monde. Renverser l'Amérique, c'est révolter les sens, leur donner des directions non fixées, non attachées, les laisser tomber au Sud et les laisser, dans la chute, se débrouiller eux-mêmes sur l'abîme qui plonge le nord et invite - il n'y aurait pas d'autre choix - à danser, comme Nietzsche, sur lui'. Danse et dessin semblent trouver ici quelque chose de commun: tous deux nous parlent d'un mouvement vers et par le sensible, du détachement des coordonnés qui les soutiennent, de la nécessité de répéter l'expérience du vide, l'expérience de l'abîme, l'expérience qu'il faut forger lorsque on a quitté ce qui se constitue à la façon des coordonnées ou à la façon d'une dialectique éternelle entre ce qui est coordonné et ce qui vient à le désarmer.

L'autonomie n'est pas pensée donc de manière identitaire, mais au niveau du sensible : l'Amérique latine se fait maintenant, avec le dessin d'une Amérique inversée, sensible face à la sensibilité même. Cela veut dire qu'on ne peut pas lire le renversement de l'Amérique avec les mêmes codes. Au contraire, il faut laisser tomber et plonger le Nord pour avoir le sentiment de l'avoir perdu et d'avoir rencontré un Sud qui ne compte pas avec des coordonnées.

11 Ainsi, avant d'être une carte, América Invertida est un dessin et même le dessin d'un projet de carte - projet qui est pourtant et toujours plus vaste, qui est dans un excès par rapport à ce que pourrait soutenir une carte. Et ce dessin n'est pas une simple affaire de 
visible mais de sensible ${ }^{10}$ : il s'agit en effet des sens, du sens des sens, des coordonnés qui fixent les sens, de ce qu'on sent, de vers où l'on sent. L'inversion dépasse même l'image de l'Amérique, car l'inversion nous dit que la carte est avant tout un dessin, une articulation sensible. C'est-à-dire que ce qu'on voit s'inverser, c'est le contrat existant entre la carte, le dessin et l'orientation : tous séparés, la défiguration de la carte est aussi la défiguration de l'espace et la désorientation de l'expérience comme une rencontre imprécise entre le sensible et leurs voies de déploiement, comme retour du destin - le Sud - sans destin, comme séparation infinie entre le destin, le lieu, la carte et le sens, séparation qui anticipe l'anticipation même de cet artefact qu'est une carte. C'est la fin d'un territoire. Lorsque le Sud remplace le nord, on avait perdu le destin. On avait gagné un destin sans destin : le Sud.

L'América Invertida n'est donc pas une révolte dans les affaires cartographiques. Moins encore est-elle une aventure créative dans l'histoire des cartes. Elle est plutôt une sorte de détachement du sensible d'un territoire qui ne se sent plus; le détachement du territoire même.

Cette anticipation du déploiement sensible face au territoire, on la trouve chez Gilles Deleuze. Chez lui, les agencements territoriaux naissent à partir de l'apparition de certaines organisations de sons, la ritournelle. Certains accouplements, certains ensembles de sons, certaines répétitions temporelles et mélodiques donnent l'image des limites fixes à travers l'établissement sonore d'une répétition, noyau mélodique de la logique et du territoire. Précisément la possibilité de la fixation des limites naît avec l'apparition de la ritournelle. On peut donc dire: toute carte est anticipée par une ritournelle; lorsqu'on trace les traits d'une carte, on répète la chanson, tout comme le film d'Alain Resnais On connait la chanson - auquel Bernard Stiegler consacre des analyses ${ }^{11}-$, là où la vie des citoyennes est toujours décrite par la répétition anticipatrice de chansons. Mais à l'inverse, le Sud n'est pas une chanson connue, il n'est pas encore une chanson et il ne le sera jamais peut-être. Mais il faut tout de même l'écouter comme tel, sans chanson connue: c'est cela le renversement de Joaquín Torres García.

14 La ritournelle fixe des coordonnées. Introduit un ordre, met un ordre dans tout ce qui arrive comme terre-sans-coordonnés. Chez Deleuze, cet ordre fixé, encore une fois, est fixé par rapport au son. Il s'agit d'un ordre, d'un mur, d'un mur territorial où « les composantes vocales, sonores, sont très importantes: un mur du son, en tout cas un mur dont certaines briques sont sonores ${ }^{12}$. Ces briques constituent les fondements du mur : ce n'est qu'à partir de la sonorisation répétée de la ritournelle que commence la construction du mur, la levée de tous les types de frontières qui dessinent le territoire.

On a souvent souligné le rôle de la ritournelle: elle est territoriale, c'est un agencement territorial. Les chants d'oiseaux : l'oiseau qui chante marque ainsi son territoire... Les modes grecs, les rythmes hindous, sont eux-mêmes territoriaux, provinciaux, régionaux. La ritournelle peut prendre d'autres fonctions, amoureuse, professionnelle ou sociale, liturgique ou cosmique: elle emporte toujours de la terre avec soi, elle a pour concomitant une terre, même spirituelle, elle est en rapport essentiel avec un Natal, un Natif. Un « nome » musical est un petit air, une formule mélodique qui se propose à la reconnaissance, et restera l'assise ou le sol de la polyphonie (cantus firmus). Le nomos comme loi coutumière et non écrite est inséparable d'une distribution d'espace, d'une distribution dans l'espace, par là il est ethos, mais l'ethos est aussi bien la Demeure. Et tantôt l'on va du chaos à un seuil d'agencement territorial : composantes directionnelles, infra-agencement. Tantôt l'on organise l'agencement: composantes dimensionnelles, intra-agencement. 
Tantôt l'on sort de l'agencement territorial, vers d'autres agencements, ou encore ailleurs : inter-agencement, composantes de passage ou même de fuite. Et les trois ensembles. Forces du chaos, forces terrestres, forces cosmiques : tout cela s'affronte et concourt dans la ritournelle ${ }^{13}$.

On peut donc déduire qu'il y a une disposition musicale dans toute construction, dans toute décision, dans tout enjeu tel que Nietzsche l'avait décrit à propos de Schiller dans l'Origine de la tragédie. En trouvant certaines associations des sons, la ritournelle fait donc apparaître un dessin comprimé du territoire, un dessin qui va dessiner le territoire, qui va faire le plan du monde, d'une cartographie qui nous donne une façon de voir, de comprendre et un début d'organisation d'un monde auparavant désordonné, ce que Deleuze appelle le chaos. La musique protège.

Un enfant dans le noir, saisi par la peur, se rassure en chantonnant. Il marche, s'arrête au gré de sa chanson. Perdu, il s'abrite comme il peut, ou s'oriente tant bien que mal avec sa petite chanson. Celle-ci est comme l'esquisse d'un centre stable et calme, stabilisant et calmant, au sein du chaos. Il se peut que l'enfant saute en même temps qu'il chante, il accélère ou ralentit son allure; mais c'est déjà la chanson qui est elle-même un saut : elle saute du chaos à un début d'ordre dans le chaos, elle risque aussi de se disloquer à chaque instant. Il y a toujours une sonorité dans le fil d'Ariane. Ou bien le chant d'Orphée ${ }^{14}$.

Platon savait déjà que le contrôle des types de musique qu'on joue dans la cité est indispensable pour la formation de la République. Aujourd'hui les choses n'ont pas changé : l'industrie musicale réduit la musique à des limites très étroites, en produisant des vides musicaux vers lesquels les plus jeunes accourent en cherchant précisément un territoire. Platon pensait la musique comme répétition et reflet de l'harmonie du cosmos : répétition de la répétition, la musique ordonne les sens du ciel dans l'âme, elle territorialise le ciel, lui donne un nord. Mais la variante deleuzienne est plus subtile : à l'inverse, la ritournelle sera qui aura projeté un ciel ordonné, dirigé, plein du sens. Et la musique sera la répétition de la ritournelle, la répétition de cette répétition - la musique avant le ciel ou, plutôt, comme le ciel : des virtuels, deux virtuels sous la même répétition. Chez Platon - et pour Deleuze ou avec Deleuze - le ciel devient une codification ou une transcendentalisation de la ritournelle, à la façon de la machine despotique barbare dont Deleuze parle dans l'Anti- $E d i p e^{15}$. Là, la pensée, le sensible, perdent la ritournelle, agissent sans avoir de nouvelles de la ritournelle.

Si nous envisageons une pensée sur la musique à partir de sa séparation d'avec le son, la musique, se réveillerait elle-même comme une sorte de mouvement ou de cinétique. Et elle peut tomber parfois dans un caractère conservateur, parfois dans un caractère non conservateur. Elle peut s'inscrire dans une sorte de logique œdipienne ou non œdipienne. Expliquons-nous : cette ritournelle, ou plutôt, le sujet qui naît avec cette ritournelle, le petit enfant et son sentiment de sécurité, c'est-à-dire le sujet apparaissant avec cette répétition sonore et rythmique, peut soit exercer l'action de se protéger et de conserver le nouveau territoire, les nouvelles logiques et associations découvertes, soit il peut faire l'expérience continue d'ouvrir son propre territoire, à savoir, la répétition différente de la ritournelle, le petit changement introduit chaque fois qu'elle tourne ou qu'elle roule. Ainsi, chez Deleuze :

1. la fixation éternelle d'une ritournelle, cette image qui n'est rien d'autre que la peur de retourner à l'état où se trouvait le petit enfant avant le calme délivrée par la ritournelle, ne pourrait naître que, justement, de la peur, d'une terreur passionnée de perdre les cycles, de sentir venir l'anarchie sonore et la multiplicité de sens sans Nord qui hante toutes les formes 
de la conscience et de l'inconscience. Il n'est pas étrange que la musique soit, chez Platon, liée aux gardiens ${ }^{16}$.

2. La qualité de la ritournelle est sa multiplicité. Entre deux ritournelles il n'y a pas du vide, il y a la transformation mutuelle, l'affectation de l'une dans l'autre et de l'autre dans l'une. Cela signifie : la ritournelle est multiple, mais elle n'est pas coupée - «On sort de chez soi au fil d'une chansonnette ${ }^{17}$. La ritournelle est un ensemble infini de cycles et répétitions qui se trouvent et s'affectent, tout en changeant leurs propres rythmes et leurs propres formes dans une interaction infinie qui n'arrête pas. La ritournelle ne peut pas être comptée, car elle est à la fois un moment composé par d'infinies ritournelles et un moment qui compose une ritournelle plus vaste, sans qu'on puisse identifier sa position, sans finalement qu'on puisse compter sur une cartographie des ritournelles - la ritournelle même est la condition de toute cartographie, ce qui transforme la cartographie en enquête dessinant et colorant des intensités rythmiques, des intensités répétitives, des places de reconnaissance répétitive.

Nous proposons ici une troisième possibilité: l'existence d'un espace non lié, sans ritournelle à l'origine. Pour cela, ce qu'il faut souligner, c'est la place même des sons. Si nous nous concentrons sur la situation du petit enfant avant l'arrivée de la ritournelle, on trouve qu'il y a des sons partout. Il est impossible que tous entrent dans le cycle de cette ritournelle. Celle-ci a dû laisser des sons hors son propre cycle, c'est-à-dire qu'il y a des sons qui subsistent comme restes, qui entourent, qui échappent et même - ce qui n'est pas possible avec Deleuze - qui n'ont aucun lien avec la ritournelle. Si cette machine immense d'infinies ritournelles qui est la ritournelle-même ne cesse pas de tourner et rouler, comme la Terre et les planètes, elle ne laisse pas non plus se créer des sons sans ritournelle, c'est-à-dire des sons sans lien comme ceux que voulait toujours écouter quelqu'un comme John Cage ${ }^{18}$.

Il faut penser qu'un territoire repose sur un territoire plus vaste, un territoire non codifié, un territoire multiple, un territoire qui ne porte pas le nom de territoire, tout en laissant deviner quelque chose qui peut aller au-delà de l'espace même et que Deleuze avec Guattari appellent le chaosmos. Mais celui-ci n'est pas un espace vide qui accueille le déploiement de la ritournelle - le lieu où celle-ci se poserait : il est plutôt la ritournelle à l'infini, au-delà de ce qu'on peut compter depuis un territoire déterminé. Le territoire est un moment, un point où le cosmos semble une fixation, une forme, un lieu. De cette manière, le territoire ne s'oppose pas au cosmos; ils communiquent, ils sont liés. En termes deleuziens, entre le territoire et le cosmos, il y a des flux. Autrement dit, la ritournelle n'est pas séparée, elle-même sort de soi-même à la manière de la ritournelle, car si l'on pensait la ritournelle comme castration - comme coupure de soi par rapport à un autre -, comme đEdipe, le territoire resterait fermé à lui-même, sans possibilité de s'ouvrir au cosmos, au-delà de soi. Cela serait la ritournelle pensée à partir de sa pure répétition, sans différence, comme la pulsion de mort. Si l'on rappelle que le son est la propagation des vagues à partir de la vibration des corps, on pourra penser que le son est la pulsion de mort elle-même. Un battement de mort répandrait les sons d'une terre (et cela résonne dans l'idée de résonance sinistre de l'artiste sonore anglais David Toop ${ }^{19}$ ).

Chez Deleuze, au contraire, la ritournelle ne doit pas être pensée comme une coupure insurmontable entre le territoire et le cosmos. Un chant a toujours la possibilité, dans sa répétition, de sortir vers cosmos, c'est-à-dire, de sortir de soi, de poursuivre son émission. Un chant est une émission, il persiste comme vibration; ce n'est pas la détention ni l'enveloppe des sons. La sortie est toujours ouverte, les sons se 
poursuivent en vibrant. C'est la troisième caractéristique que Deleuze donne de la ritournelle : la sortie vers le $\operatorname{cosmos}^{20}$.

21 Mais - et c'est ici qu'on trouve la troisième possibilité dont nous avons parlée - la sortie vers le cosmos proposée par Deleuze, est toujours dirigée. Le Cosmos-Chaosmos, c'est l'infinité des ritournelles. C'est l'immanence : on ne peut pas sauter, la ritournelle sera tout - et rien de plus. La sortie vers le chaosmos sera d'amener la ritournelle aux destins les plus impensés mais toujours immanents, d'une certaine manière déjà répétés en elle-même. Il s'agit de la plasticité de la ritournelle, de son élasticité infinie, de la plasticité qui est le territoire, l'univers, tout. Sortir vers le cosmos, c'est commencer à dominer la plasticité, l'élasticité, la puissance de transformation. Puissance toujours immanente, c'est-à-dire sans intervalles, sans espaces vides. La case vide dont parlait Deleuze $^{21}$, c'était en fait la puissance de transformation même, ce qui dans ou dès le territoire reste comme principe de mobilité, comme ritournelle avancée ou retardée, comme temporalité ou vitesse qui tente le territoire, comme espace non dessiné par la cartographie fidèle au territoire. C'est l'espace où la plasticité peut s'étendre sans se détacher d'elle-même, la condition de la transformation infinie. Mais elle - la case vide - n'a rien à voir avec un lieu ou un espace vide comme tel, c'est-à-dire déconnecté, délié, tout à fait étranger, coupé par une distance irréductible, comme s'il y avait deux ritournelles dont la rencontre et le contact mutuel seraientt impossibles.

C'est ici que l'espace de sons non liés dont nous avons parlé plus haut revient encore une fois : il apparaît dans la philosophie de Gilles Deleuze comme un moment surmonté à jamais. On pourrait dire qu'il fonctionne comme une image mythique, car, à vrai dire, il n'existe pas, il n'est pas possible. Lorsque le petit enfant se protège avec la naissance de la ritournelle, Deleuze semble nous dire que la ritournelle était toujours là, qu'il fallait attendre pour sentir rouler la répétition et faire advenir ce qui était toujours là, la ritournelle: il n'y a pas de pas d'un chaos vide à la ritournelle, mais le triomphe éternel de la ritournelle sur ce qui apparaît non lié, vide. Celui-ci, c'est le mythe, le mythe qui prend forme, dans la philosophie de Gilles Deleuze, d'un enfant qui a peur lorsqu'il est perdu dans le noir. Être-non-lié, c'est, dans ce mythe, l'obscurité, le noir. Maintenir un lien sans lien, comme deux personnes communiquant dans le vide, c'est ce qui ne peut pas arriver, non par interdiction, mais par impossibilité ontologique. Mais nous avons dit - tout en rappelant la condition sonore de la ritournelle et la formation de la terre, le chant des oiseaux portant la ritournelle et marquant le territoire - qu'une sonorité restait comme reste, que la totalité transformiste de la ritournelle (comme infini de ritournelles) laissait des restes sonores (qui n'ont pas en fait la forme de briques) qui ne s'introduisaient pas dans cette répétition infinie. Notre position donc, toute proche de celle d'Alain Badiou par exemple, c'est celle d'une multiplicité sans ritournelle ${ }^{22}$. C'est-à-dire, il faut ne pas l'oublier, que si le territoire est ce qui se forge par une délimitation sonore, il y aura toujours un territoire non lié, un territoire qui n'est pas non plus un lieu déterritorialisé, mais un espace, si vous voulez, sans lien interne et sans lien externe. Quelque chose qui n'est pas enregistré dans la ritournelle et la plasticité de ses répétitions. Il s'agit d'un lieu où le chant des oiseaux se disperse à l'infini, sans faire un territoire, s'écoute sans territoire. La brique sonore est justement le contraire de la dispersion sonore, de cette sonorité qui ne trouve pas le commencement de sa répétition, le point mythique de départ.

On le voit: si la disposition des sons est celle qui dessine le territoire, celle qui commande l'orientation du dessin de la carte, on a chez Deleuze une carte qui est un 
artefact plastique infini. L'expérimentation dessinatrice n'est justement que la possibilité d'étendre les possibilités transformistes de tout territoire, de la territorialité même, c'est-à-dire le dessin qui répète toujours sa sortie vers le cosmos.

En ce sens, toute cartographie est sa pure plasticité. La carte pourra toujours se figurer et se défigurer, territorialiser et déterritorialiser. Mais ce qui commande le dessin, l'orientation écoutante, semble chez Deleuze rester toujours à l'abri des ritournelles, comme si l'on ne pouvait pas écouter des sons non liés, de musique sans musique, des sons par hasard. On pourrait renverser la situation et nous interroger sur la possibilité de faire des cartes, de concevoir des territoires à partir d'une écoute sans ritournelles, ou de ritournelles non liées. En ce sens, le dessin d'une Amérique latine inversée, même si Joaquín Torres García assurait retrouver sa position, n'est pas encore une carte, elle ne figure pas son territoire, ce qui serait son territoire, son adresse, son orientation. On propose de le voir comme un dessin où l'orientation écoutante résiste à la ritournelle, où elle n'a pas résolu l'écoute même. Un déplacement en excès de toute ritournelle, comme si l'on avait sauté au-delà de sa plasticité. L'América Invertida ne veut pas simplement trouver l'Amérique du Sud: elle veut surtout faire de l'Amérique un lieu d'écoute.

Pour finir, Deleuze dit «Le territoire serait l'effet de l'art " ${ }^{23}$. Mais on peut penser à un art qui voudrait quitter son effet. Un territoire qui, malgré lui, revient à l'art pour se poser la question de comment vivre. Ainsi, on voit chez Torres García une séparation des causes et effets. Il ne s'agit pas de dessiner une carte, mais de diluer la carte vers le dessin, vers la puissance artistique, vers le sensible. Et il n'y a pas non plus une déterritorialisation, ce contre-effet dialectique ou élastique: il s'agit, on le répète, d'échapper à cet effet et à ce contre-effet, de se placer dans le point où la carte, ce dessin d'une Amérique latine inversée, revient à l'art. On dira que ce dessin refait un territoire. Certes, mais on ne sait pas bien où le Sud est. La question est précisément là : le nord, c'est le territoire, le principe de territorialisation et déterritorialisation, le magnétisme du jeu, la case vide. Le Sud est autre chose, une autre expérience de la terre, une expérience étrangère aux coordonnées.

\section{BIBLIOGRAPHIE}

Badiou Alain, La République de Platon, Paris, Fayard, 2012.

Deleuze Gilles, « À quoi reconnaît-on le structuralisme? » in Histoire de la philosophie VIII. Le XX siècle, François Châtelet, Paris, Hachette, 1973.

Deleuze Gilles \& Guattari Félix, Anti-〔Edipe. Capitalisme et Schizophrénie, Paris, éditions de Minuit, 1972.

Deleuze Gilles \& Guattari Félix, Mille Plateaux. Capitalisme et Schizophrénie, Paris, éditions de Minuit, 1980.

Deleuze Gilles \& Guattari Félix, Qu'est-ce que la philosophie, Paris, éditions de Minuit, 2005. 
Nietzsche Friedrich, Le gai savoir, trad. par Patrick Wolting, Paris, Flammarion, Paris, 2007.

Platon, La République, trad. par Georges Leroux, Paris, Flammarion, 2008.

Rancière Jacques, Le partage du sensible, Paris, La Fabrique, 2000.

Rancière Jacques, Béla Tarr, le temps d'après, Nantes, Caprici Éditions, 2011.

Stiegler Bernard, La technique et le temps 1. La faute d'Épiméthée, Paris, éditions Galilée, 1994.

Stiegler Bernard, De la misère symbolique 1. L'époque hyperindustrielle, Paris, éditions Galilée, 2004.

Stiegler Bernard, Économie de l'hypermatériel et psychopouvoir, Paris, éditions Mille et une Nuits, 2008.

Toop David, Sinister Resonance: The Mediumship of the Listener, London, Continuum International Publishing Group, 2010.

Torres García Joaquín, Universalismo Constructivo 1, Madrid, Alianza Editorial, 1984.

Wood Denis, Rethinking the powers of maps, New York, Gilford Press, 2010.

Wood Denis, Everything sings. Maps for a narrative Atlas, New York, Siglio Press, 2013.

\section{NOTES}

1. http://www.cuadernoscepla.cl/

2. On peut remarquer que l'auteur a choisi d'écrire nord avec une minuscule et Sud avec une majuscule. Sauf exception, nous conserverons ce principe.

3. Joaquín Torres García, Universalismo Constructivo 1, Madrid, Alianza Editorial, 1984, p. 193. Traduction par nos soins.

4. C'est par exemple la position d'André Leroi-Gourhan auquel quelqu'un comme Bernard Stiegler emprunte pour constituer sa pensée. Voir Économie de l'hypermatériel et psychopouvoir, Paris, Mille et une nuits, 2008, p. 54,64. Voir aussi La technique et le temps 1. La faute d'Épiméthée, Paris, éditions Galilée, 1994, p. 152.

5. Voir l'œuvre intitulée Map (1961) où il approfondit la couleur dans le territoire des États-Unis et morceaux de Canada et Mexico. Tout se passe comme si la carte semblait être dessinée, se diluer dans l'aquarelle afin d'émanciper un certain événement auquel la carte ne donne pas lieu.

6. Denis Wood, Everything sings. Maps for a narrative Atlas, New York, Siglio Press, 2013. Voir aussi son site internet, http://makingmaps.net/. Il a aussi écrit un livre intitulé Rethinking the powers of maps (New York, Gilford Press, 2010). Nous ne faisons pourtant pas une révision critique de sa recherche. Nous signalons tout simplement la tentative de considérer les cartes comme structures narratives, voire comme mythes qui dessinent - son site internet est plein d'expérimentations des cartes - la chorégraphie (c'est notre mot) qu'il faut représenter dans le territoire que la carte construit.

7. Jacques Rancière, Le partage du sensible, Paris, La Fabrique, 2000.

8. Afin d'éviter des confusions, précisons que nous n'affirmons pas que la carte est une extension graphique de l'idée. Nous disons plutôt qu'il y de l'idée avant le développement d'une nouvelle carte. Idée diffuse, perdue, désorientée, multiple. Cette idée provient d'une vieille carte, d'un vieil artefact de territoires. Cette carte et cet artefact avaient une idée qui les précédait, idée qui provenait aussi d'un ancien artefact de territoires. En comprimant, le jeu d'antériorités semble devenir insoutenable, fatigant. Mais la question importante, pour nous, c'est de reproduire immédiatement l'existence indépendante de l'un (la carte, la technique) par rapport à l'autre (le territoire, le style, l'esprit, le sujet, l'habitant). Et vice versa. 
9. Friedrich Nietzsche, Le gai savoir, trad. par Patrick Wotling, Paris, Flammarion, 2007, § 347.

10. Jacques Rancière dit, à propos de Bela Tarr, que le cinéma n'est pas art du visible mais un art du sensible (Béla Tarr, le temps d'après, Nantes, Caprici Éditions, 2011, p. 11).

11. Bernard Stiegler, De la misère symbolique 1. L'époque hyperindustrielle, Paris, éditions Galilée, 2004 , p. 41 sqq.

12. Gilles Deleuze \& Félix Guattari, Mille Plateaux. Capitalisme et Schizophrénie, Paris, éditions de Minuit, 1980, p. 382.

13. Ibid., p. 383-394.

14. Ibid., p. 382.

15. Gilles Deleuze \& Félix Guattari, Anti-đEdipe. Capitalisme et Schizophrénie, éditions de Minuit, Paris, 1972, chapitre III.

16. Platon, La République, Livre III. Il faut évoquer, pour être juste, la vision des gardiens - en ce qui concerne la musique et les disciplines de l'esprit - que fait Alain Badiou dans La République de Platon (Paris, Fayard, 2012), liée à l'idée d'un éternel retour des vérités et le courage qu'il faut avoir pour y habiter.

17. Gilles Deleuze \& Félix Guattari, Mille Plateaux. Capitalisme et Schizophrénie, op. cit., p. 383.

18. Il y a plusieurs endroits où l'on peut trouver cette idée fondamentale de la musique de John Cage. Voyez notamment l'entretien qui apparaît dans le film de Miroslav Sebestik, Écoute (Centre Georges Pompidou, JBA Production, SACEM, La Sept, Mikros Image, Paris, 1992).

19. David Toop, Sinister Resonance: The Mediumship of the Listener, Londres, Continuum International Publishing Group, London, 2010.

20. Gilles Deleuze \& Félix Guattari, Mille Plateaux. Capitalisme et Schizophrénie, op. cit., p. 381-383. Trois pas : l'agencement territorial, l'ouverture ou l'expansion circulaire du territoire et la sortie vers le cosmos.

21. Gilles Deleuze, «À quoi reconnaît-on le structuralisme ?» in Histoire de la philosophie VIII. Le XXe siècle, F. Châtelet, Paris, Hachette, 1973.

22. L'idée de Badiou d'une multiplicité quelconque est considérée par Deleuze et Guattari comme impossible. Gilles Deleuze \& Félix Guattari, Qu'est-ce que la philosophie, Paris, éditions de Minuit, 2005, p. 143 sqq.

23. Gilles Deleuze \& Félix Guattari, Mille Plateaux. Capitalisme et Schizophrénie, op. cit., p. 388.

\section{RÉSUMÉS}

Cet article est une réflexion sur certaines conséquences philosophiques et esthétiques d'une pensée critique des idées deleuziennes de ritournelle et de territorialité. Il développe les rapports entre art et cartographie, entre sonorité, dessin et construction des territoires. Son idée principale vise à renverser la direction qui s'établit généralement entre l'art et le territoire, tout en avançant l'hypothèse d'un art qui ne serait pas lié aux motifs de la territorialisation et de la déterritorialisation.

INDEX

Mots-clés : carte, ritournelle, territoire, son, art, orientation 
AUTEUR

\section{GUSTAVO CELEDÓN BÓRQUEZ}

Chercheur de l'Universidad de Valparaíso et Laboratoire des logiques contemporaines de la philosophie, Paris 8, gustavoceledon@gmail.com 